\title{
Geometric Hardy inequalities for the sub-elliptic Laplacian on convex domains in the Heisenberg group
}

\author{
Simon Larson ${ }^{1}$
}

Received: 1 April 2016 / Accepted: 13 April 2016 / Published online: 28 April 2016

(C) The Author(s) 2016. This article is published with open access at Springerlink.com

Abstract We prove geometric $L^{p}$ versions of Hardy's inequality for the sub-elliptic Laplacian on convex domains $\Omega$ in the Heisenberg group $\mathbb{H}^{n}$, where convex is meant in the Euclidean sense. When $p=2$ and $\Omega$ is the half-space given by $\langle\xi, v\rangle>d$ this generalizes an inequality previously obtained by Luan and Yang. For such $p$ and $\Omega$ the inequality is sharp and takes the form

$$
\int_{\Omega}\left|\nabla_{\mathbb{H}^{n}} u\right|^{2} d \xi \geq \frac{1}{4} \int_{\Omega} \sum_{i=1}^{n} \frac{\left\langle X_{i}(\xi), v\right\rangle^{2}+\left\langle Y_{i}(\xi), v\right\rangle^{2}}{\operatorname{dist}(\xi, \partial \Omega)^{2}}|u|^{2} d \xi
$$

where $\operatorname{dist}(\cdot, \partial \Omega)$ denotes the Euclidean distance from $\partial \Omega$.

Mathematics Subject Classification 35A23 - 35H20

\section{Introduction}

In [12] Luan and Yang prove the Hardy inequality

$$
\int_{\mathbb{H}_{+}^{n}}\left|\nabla_{\mathbb{H}^{n}} u\right|^{2} d \xi \geq \int_{\mathbb{H}_{+}^{n}} \frac{|x|^{2}+|y|^{2}}{t^{2}}|u|^{2} d \xi
$$

Communicated by Ari Laptev.

Simon Larson

simla@math.kth.se

1 Department of Mathematics, Royal Institute of Technology, 10044 Stockholm, Sweden 
where an element $\xi \in \mathbb{H}^{n}$ is written as $\xi=(x, y, t)$, with $x, y \in \mathbb{R}^{n}$ and $t \in \mathbb{R}$, and $\mathbb{H}_{+}^{n}:=\left\{\xi \in \mathbb{H}^{n}: t>0\right\}$. In this paper we provide a different proof of this inequality, generalize it to any half-space of $\mathbb{H}^{n}$ and use it to obtain a weighted geometric Hardy inequality on a convex domain $\Omega$, where convex is meant in the Euclidean sense. The weight that appears in our results is in some sense a natural sub-elliptic weighting of the Euclidean distance and is closely related to distances studied in $[14,16]$.

We begin with a short introduction providing the basic definitions, notation and background necessary for the sequel.

The $n$-dimensional Heisenberg group, which we denote by $\mathbb{H}^{n}$, may be described as the set $\mathbb{R}^{2 n+1}$ equipped with the group law

$$
\hat{\xi} \circ \tilde{\xi}:=\left(\hat{x}+\tilde{x}, \hat{y}+\tilde{y}, \hat{t}+\tilde{t}+2 \sum_{i=1}^{n}\left(\tilde{x}_{i} \hat{y}_{i}-\hat{x}_{i} \tilde{y}_{i}\right)\right)
$$

where we use the notation $\xi=\left(x_{1}, \ldots, x_{n}, y_{1}, \ldots, y_{n}, t\right)=(x, y, t) \in \mathbb{R}^{2 n+1}$. The inverse element of $\xi$, with respect to the group law, is denoted by $\xi^{-1}$ and we note that $\xi^{-1}=-\xi$. The group law induces the following dilation operation

$$
\delta_{\lambda}(\xi):=\left(\lambda x, \lambda y, \lambda^{2} t\right) \text { for } \lambda>0 .
$$

The Lie algebra of left-invariant vector fields on $\mathbb{H}^{n}$ is spanned by

$$
X_{i}:=\frac{\partial}{\partial x_{i}}+2 y_{i} \frac{\partial}{\partial t} \text { and } Y_{i}:=\frac{\partial}{\partial y_{i}}-2 x_{i} \frac{\partial}{\partial t},
$$

for $1 \leq i \leq n$, together with their commutators. The only non-zero commutators are

$$
\left[X_{i}, Y_{i}\right]=-4 \frac{\partial}{\partial t}
$$

We also define the associated gradient $\nabla_{\mathbb{H}^{n}}:=\left(X_{1}, \ldots, X_{n}, Y_{1}, \ldots, Y_{n}\right)$ and the Heisenberg Laplacian $\Delta_{\mathbb{H}^{n}}$ on $\mathbb{H}^{n}$, formally given by $\Delta_{\mathbb{H}^{n}}:=\sum_{i=1}^{n} X_{i}^{2}+Y_{i}^{2}$. The collection of vector fields $\left\{X_{i}, Y_{i}: 1 \leq i \leq n\right\}$ satisfies the Hörmander finite rank condition:

$$
\operatorname{Rank} \operatorname{Lie}\left[X_{1}, \ldots, X_{n}, Y_{1}, \ldots, Y_{n}\right]=2 n+1 \text {. }
$$

Thus the Heisenberg Laplacian is a second order hypoelliptic differential operator [10].

We call a Lipschitz curve $\gamma: I \subset \mathbb{R} \rightarrow \mathbb{H}^{n}$ horizontal if its tangent at almost every $\tau \in I$ is spanned by the $X_{i}$ and $Y_{i}$, that is, for a.e. $\tau \in I$ there exist $a, b \in \mathbb{R}^{n}$ such that

$$
\gamma^{\prime}(\tau)=\sum_{i=1}^{n} a_{i} X_{i}(\gamma(\tau))+b_{i} Y_{i}(\gamma(\tau))
$$


We denote the set of all horizontal curves $\gamma: I \rightarrow \mathbb{H}^{n}$ by $\mathcal{S}_{\mathbb{H}}(I)$ and for a given $\gamma \in \mathcal{S}_{\mathbb{H}^{n}}(I)$ we define its length $l(\gamma)$ as

$$
l(\gamma):=\int_{I}\left(|a(\tau)|^{2}+|b(\tau)|^{2}\right)^{1 / 2} d \tau .
$$

By the accessibility theorem of Chow and Rashevsky any pair of points $\xi_{0}, \xi_{1} \in \Omega$ where $\Omega$ is an open connected subset of $\mathbb{H}^{n}$ can be joined by a horizontal curve $\gamma:[0,1] \rightarrow \mathbb{H}^{n}$ of finite length (see $[5,15]$ ).

The Carnot-Carathéodory distance $\delta_{c c}$ on $\mathbb{H}^{n}$ is defined as

$$
\delta_{c c}\left(\xi_{0}, \xi_{1}\right):=\inf \left\{l(\gamma): \gamma \in \mathcal{S}_{\mathbb{H}^{n}}([0,1]), \gamma(0)=\xi_{0}, \gamma(1)=\xi_{1}\right\}
$$

The Carnot-Carathéodory distance is not the only distance that has a natural connection to $\mathbb{H}^{n}$. A second distance that arises naturally when considering the fundamental solution of $\Delta_{\mathbb{H}^{n}}$ is the Kaplan distance (see [9]):

$$
\delta_{K}\left(\xi_{0}, \xi_{1}\right):=\rho\left(\xi_{1}^{-1} \circ \xi_{0}\right)
$$

where $\rho$ is the Kaplan gauge on $\mathbb{H}^{n}$ defined by

$$
\rho(\xi):=\left(\left(|x|^{2}+|y|^{2}\right)^{2}+4 t^{2}\right)^{1 / 4} .
$$

It turns out that the two distance functions above are bi-Lipschitz equivalent, that is, there exists a constant $C>0$ such that for all $\xi^{0}, \xi^{1} \in \mathbb{H}^{n}$ we have that

$$
C^{-1} \delta_{K}\left(\xi_{0}, \xi_{1}\right) \leq \delta_{c c}\left(\xi_{0}, \xi_{1}\right) \leq C \delta_{K}\left(\xi_{0}, \xi_{1}\right)
$$

Let $\mathcal{M} \subset \mathbb{H}^{n}$ be a $2 n$-dimensional $C^{1}$ manifold. We call a point $\xi_{0} \in \mathcal{M}$ a characteristic point of $\mathcal{M}$ if the tangent space $T_{\xi_{0}} \mathcal{M}$ is spanned by $\left\{X_{i}\left(\xi_{0}\right),, Y_{i}\left(\xi_{0}\right)\right.$ : $1 \leq i \leq n\}$.

Even though both $\delta_{c c}$ and $\delta_{K}$ appear naturally when considering the geometric structure of $\mathbb{H}^{n}$ these distances can be rather difficult to work with, see for instance the work of Arcozzi and Ferrari [1,2]. Extra difficulties arise when studying the behaviour of the distance to a hypersurface $\mathcal{M}$ close to one of its characteristic points.

In what follows we will be interested in inequalities of the form

$$
\int_{\Omega}\left|\nabla_{\mathbb{H}^{n}} u(\xi)\right|^{p} d \xi \geq C \int_{\Omega} \frac{|u(\xi)|^{p}}{\rho(\xi, \partial \Omega)^{p}} d \xi,
$$

where $\Omega \subset \mathbb{H}^{n}, p \geq 2$ and $\rho$ is some, possibly weighted, distance from $\xi$ to the boundary of $\Omega$. In the Euclidean setting, with $\nabla_{\mathbb{H}^{n}}$ replaced by the usual gradient and $\rho$ by the Euclidean distance, such inequalities have a long history and wide range of applications (see, for instance, $[4,8,13]$ ).

In the setting of the Heisenberg group results of this kind have been obtained through methods based on sub-elliptic capacity and Fefferman-Phong inequalities. 
In [7] the authors provide sharp conditions on the triple $\Omega, \rho$ and $C$ for the validity of (2). However, the results obtained in [7] are of a rather non-explicit nature and what they say in a specific setting is not very approachable.

One of the obstacles in proving inequalities of the form (2) on domains in $\mathbb{H}^{n}$ is that the natural distances on $\mathbb{H}^{n}\left(\delta_{c c}, \delta_{K}\right)$ are rather difficult to work with. If $\Omega=\mathbb{H}^{n} \backslash\left\{\xi_{0}\right\}$ and $\rho$ is the Carnot-Carathéodory or Kaplan distance to the point $\xi_{0} \in \mathbb{H}^{n}$ inequalities of this form have been studied in a several articles (see, for instance, $[6,9,11,17]$ ). However, when $\partial \Omega$ is a more complicated set the problem becomes more difficult. Results concerning the behaviour of the distance from sets and a detailed analysis of the problems arising can be found in work by Arcozzi and Ferrari [1,2].

In this article we begin by generalizing (1) to the case where $\Omega$ is an arbitrary half-space of $\mathbb{H}^{n}$. The proof given here differs from that given in [12] and contains their result as a special case. Moreover, from our proof of (1) and the corresponding generalizations we are able to apply a standard argument and find $L^{p}$ versions of the inequalities.

In Sect. 3, we combine our inequalities for half-spaces with a method used by Avkhadiev in the Euclidean setting [3] to obtain an inequality of the form (2) for convex domains in $\mathbb{H}^{n}$, here convex is meant in the Euclidean sense, and with $\rho$ being a weighted Euclidean distance. More specifically we have that

$$
\frac{1}{\rho(\xi)^{p}}=\sum_{i=1}^{n} \frac{\left|\left\langle X_{i}(\xi), v(\xi)\right\rangle\right|^{p}+\left|\left\langle Y_{i}(\xi), v(\xi)\right\rangle\right|^{p}}{\operatorname{dist}(\xi, \partial \Omega)^{p}}
$$

where $p \geq 2$, $\operatorname{dist}(\cdot, \partial \Omega)$ denotes the Euclidean distance to the boundary of $\Omega$ and $\nu(\xi) \in \mathbb{S}^{2 n}$ is such that $\xi+\operatorname{dist}(\xi, \partial \Omega) \nu(\xi) \in \partial \Omega$.

\section{Hardy inequalities on half-spaces of $\mathbb{H}^{n}$}

For $v \in \mathbb{S}^{2 n}$ and $d \in \mathbb{R}$ let $\Pi_{\nu, d}$ be the hyperplane in $\mathbb{H}^{n}$ defined by the equation $\langle\xi, v\rangle=d$. Correspondingly, let $\Pi_{v, d}^{+}$be the half-space of $\mathbb{H}^{n}$ where $\langle\xi, v\rangle>d$.

Theorem 2.1 Let $u \in C_{0}^{\infty}\left(\Pi_{v, d}^{+}\right)$. Then the following inequality holds

$$
\int_{\Pi_{v, d}^{+}}\left|\nabla_{\mathbb{H}^{n}} u\right|^{2} d \xi \geq \frac{1}{4} \int_{\Pi_{v, d}^{+}} \sum_{i=1}^{n} \frac{\left\langle X_{i}(\xi), v\right\rangle^{2}+\left\langle Y_{i}(\xi), v\right\rangle^{2}}{\operatorname{dist}\left(\xi, \partial \Pi_{\nu, d}^{+}\right)^{2}}|u|^{2} d \xi
$$

By choosing $v$ to be the unit vector in the $t$ direction and $d$ to be zero the above theorem reduces to (1).

In the case of a half-space it was pointed out to us by Ruszkowski that outside a certain cone the weighted distance appearing in Theorem 2.1 is comparable to the Carnot-Carathéodory distance on the Heisenberg group. In fact, the weighted distance coincides with a reduced version of the Carnot-Carathéodory distance [14,16], namely

$$
w(\xi, \partial \Omega):=\inf \left\{\delta_{c c}(\xi, \hat{\xi}): \hat{\xi} \in \partial \Omega \cap \operatorname{Span}\left(X_{i}(\xi), Y_{i}(\xi): 1 \leq i \leq n\right)\right\}
$$


For results concerning this reduced distance and Hardy inequalities closely related to those obtained here we refer to [14] and an upcoming article by Ruszkowski [16].

We proceed by providing a factorization-type proof of the above theorem and also sketch how to obtain the same statement from (1) through a simple translation argument. The second argument has the slight advantage that it gives a geometric interpretation of the weight appearing in the inequality, but most importantly it simplifies the proof that (3) is sharp. However, later in the article we will need the calculations performed in our first proof.

Proof of Theorem 2.1 The inequality is obtained by a simple factorization argument and the optimization of a parameter $\alpha$. For $u \in C_{0}^{\infty}\left(\Pi_{v, d}^{+}\right)$and any $V \in H^{1}\left(\Pi_{v, d}^{+} ; \mathbb{R}^{2 n}\right)$ with components $\left(V_{1}, \ldots, V_{2 n}\right)$ we have that

$$
\begin{aligned}
0 & \leq \int_{\Pi_{v, d}^{+}}\left|\left(\nabla_{\mathbb{H}^{n}}+\alpha V\right) u\right|^{2} d \xi \\
& =\sum_{i=1}^{n} \int_{\Pi_{v, d}^{+}}\left(\left|\left(X_{i}+\alpha V_{i}\right) u\right|^{2}+\left|\left(Y_{i}+\alpha V_{n+i}\right) u\right|^{2}\right) d \xi \\
& =\sum_{i=1}^{n} \int_{\Pi_{v, d}^{+}}\left(\left|X_{i} u\right|^{2}+\left|Y_{i} u\right|^{2}-\alpha|u|^{2}\left(X_{i}\left(V_{i}\right)+Y_{i}\left(V_{n+i}\right)\right)+\alpha^{2}|u|^{2}\left(V_{i}^{2}+V_{n+i}^{2}\right)\right) d \xi
\end{aligned}
$$

where the last equality is obtained by partial integration and the fact that $u$ has compact support.

Rearranging the terms one finds the following inequality

$$
\int_{\Pi_{v, d}^{+}}\left|\nabla_{\mathbb{H}^{n}} u\right|^{2} \geq \sum_{i=1}^{n} \int_{\Pi_{v, d}^{+}} \alpha|u|^{2}\left(X_{i}\left(V_{i}\right)+Y_{i}\left(V_{n+i}\right)-\alpha\left(V_{i}^{2}+V_{n+i}^{2}\right)\right) d \xi
$$

We now choose the components of $V$ as

$$
\begin{aligned}
V_{i}(\xi) & =\frac{\left\langle X_{i}(\xi), v\right\rangle}{\operatorname{dist}\left(\xi, \partial \Pi_{v, d}^{+}\right)}=\frac{\left\langle X_{i}(\xi), v\right\rangle}{\langle\xi, v\rangle-d} \\
V_{n+i}(\xi) & =\frac{\left\langle Y_{i}(\xi), v\right\rangle}{\operatorname{dist}\left(\xi, \partial \Pi_{v, d}^{+}\right)}=\frac{\left\langle Y_{i}(\xi), v\right\rangle}{\langle\xi, v\rangle-d} .
\end{aligned}
$$

A simple calculation gives us that

$$
X_{i}\left(V_{i}\right)(\xi)=-\frac{\left\langle X_{i}(\xi), v\right\rangle^{2}}{\operatorname{dist}\left(\xi, \partial \Pi_{v, d}^{+}\right)^{2}} \quad \text { and } \quad Y_{i}\left(V_{n+i}\right)(\xi)=-\frac{\left\langle Y_{i}(\xi), v\right\rangle^{2}}{\operatorname{dist}\left(\xi, \partial \Pi_{v, d}^{+}\right)^{2}}
$$


Inserting into equation (4) we find that

$$
\int_{\Pi_{v, d}^{+}}\left|\nabla_{\mathbb{H}^{n}} u\right|^{2} \geq-\alpha(1+\alpha) \int_{\Pi_{v, d}^{+}} \sum_{i=1}^{n} \frac{\left\langle X_{i}(\xi), v\right\rangle^{2}+\left\langle Y_{i}(\xi), v\right\rangle^{2}}{\operatorname{dist}\left(\xi, \partial \Pi_{v, d}^{+}\right)^{2}}|u|^{2} d \xi
$$

Choosing $\alpha$ to maximize $-\alpha(1+\alpha)$ completes the proof.

As mentioned above the theorem admits a second proof through a translation argument, and we proceed by sketching this alternative proof. The main reason for including this is that it reduces the proof of sharpness of (3) to considering a given half-space, but it also gives a second geometric interpretation of the weight appearing in the theorem.

We sketch the proof only in the case of $\mathbb{H}^{1}$. The ideas translate without change to higher dimension but the geometry of the argument is more transparent in the case $n=1$. For simplicity we will also only deal with the case $d=0$, i.e. a plane passing through the origin.

For $v=\left(v_{x}, v_{y}, v_{t}\right) \in \mathbb{S}^{2}$, let $\Pi_{v}$ be the plane in $\mathbb{H}^{1}$ defined by the equation $\langle\xi, v\rangle=0$. If $v_{t} \neq 0$ we can find a $\xi^{0} \in \Pi_{v}$ such that $\xi^{0}$ is a characteristic point of $\Pi_{\nu}$. This reduces to solving the following system of equations:

$$
\left\{\begin{array}{c}
\langle X(\xi), v\rangle=0 \\
\langle Y(\xi), v\rangle=0 \\
\langle\xi, v\rangle=0
\end{array}\right.
$$

From the first two equations we find that

$$
x=\frac{v_{y}}{2 v_{t}} \text { and } y=-\frac{v_{x}}{2 v_{t}}
$$

which combined with the third equation gives the solution

$$
\xi^{0}=\frac{1}{2 v_{t}}\left(\begin{array}{c}
v_{y} \\
-v_{x} \\
0
\end{array}\right)
$$

Applying a change of variables given by left translation by $-\xi^{0}$ and using the leftinvariance of $X$ and $Y$ reduces the left-hand side of inequality (3) to the case of $\Pi_{v}=\left\{\xi \in \mathbb{H}^{1}: t=0\right\}$. Thus we may apply (1). Changing back variables the right-hand side becomes, after some algebraic manipulations, the desired expression. For arbitrary non-vertical planes $\left(v_{t} \neq 0\right)$ the argument goes through without any substantial change. For vertical planes $\left(v_{t}=0\right)$ the result can be found through a simple limiting process.

In the case treated by Luan and Yang the term $|x|^{2}+|y|^{2}$ has a clear interpretation as the square of the Euclidean distance to the centre of $\mathbb{H}^{1}$, which here actually coincides with the Carnot-Carathéodory distance. This is precisely the distance from $\xi$ to the subspace of $\mathbb{H}^{1}$ consisting of points where $v$ is orthogonal to both $X$ and $Y$. 
The translation argument above then provides the interpretation that the weight corresponds to the Carnot-Carathéodory distance from the subspace where the $X_{i}$ and $Y_{i}$ span a hyperplane which is parallel to $\Pi_{v}$, multiplied by a factor corresponding to how "tilted" $\Pi_{v}$ is. If we let $\mathcal{H}_{v}$ denote the subspace of $\mathbb{H}^{1}$ given by $\left\{\xi \in \mathbb{H}^{1}\right.$ : $X(\xi) \perp v, Y(\xi) \perp v\}$ one finds that

$$
\frac{\langle X(\xi), v\rangle^{2}+\langle Y(\xi), v\rangle^{2}}{4}=v_{t}^{2} \delta_{c c}^{2}\left(\xi, \mathcal{H}_{v}\right) .
$$

One should note that this weight behaves well with respect to both $\xi$ and $v$. In particular, if we let $v_{t}$ tend to zero this converges to $1 / 4$. As this leads to a rather surprising invariance of the Hardy inequality with respect to any choice of vertical plane we state this as a corollary.

Corollary 2.2 Let $u \in C_{0}^{\infty}\left(\Pi_{v, d}^{+}\right)$with $v_{t}=0$. Then the following inequality holds

$$
\int_{\Pi_{v, d}^{+}}\left|\nabla_{\mathbb{H}^{n}} u\right|^{2} d \xi \geq \frac{1}{4} \int_{\Pi_{v, d}^{+}} \frac{|u|^{2}}{\operatorname{dist}\left(\xi, \partial \Pi_{v, d}^{+}\right)^{2}} d \xi
$$

With the above translation argument in hand we see that to prove the sharpness (3) it suffices to consider a given pair of $v \in \mathbb{S}^{2 n}$ and $d \in \mathbb{R}$. Thus we restrict our attention to the case $\Pi_{v, d}^{+}=\left\{(x, y, t) \in \mathbb{H}^{n}: t \geq 0\right\}$, that is, $v=(0, \ldots, 0,1)$ and $d=0$. We will also make use of the identity

$$
\nabla_{\mathbb{H}^{n}} u=\nabla^{\prime} u+2 \Lambda \xi^{\prime} \frac{\partial u}{\partial t}
$$

where $\xi^{\prime}=(x, y), \nabla^{\prime}$ denotes the gradient in $\mathbb{R}^{2 n}$ acting in the $\xi^{\prime}$ variables and $\Lambda$ is the skew symmetric matrix

$$
\left(\begin{array}{cc}
0 & I_{n} \\
-I_{n} & 0
\end{array}\right)
$$

It follows that

$$
\begin{aligned}
\left|\nabla_{\mathbb{H}^{n}} u\right|^{2} & =\left\langle\nabla^{\prime} u+2 \Lambda \xi^{\prime} \frac{\partial u}{\partial t}, \nabla^{\prime} u+2 \Lambda \xi^{\prime} \frac{\partial u}{\partial t}\right\rangle \\
& =\left|\nabla^{\prime} u\right|^{2}+4 \frac{\partial u}{\partial t}\left\langle\Lambda \xi^{\prime}, \nabla^{\prime} u\right\rangle+4\left|\Lambda \xi^{\prime}\right|^{2}\left|\frac{\partial u}{\partial t}\right|^{2} \\
& =\left|\nabla^{\prime} u\right|^{2}+4 \frac{\partial u}{\partial t}\left\langle\Lambda \xi^{\prime}, \nabla^{\prime} u\right\rangle+4\left|\xi^{\prime}\right|^{2}\left|\frac{\partial u}{\partial t}\right|^{2}
\end{aligned}
$$

The sharpness of (3) now follows from a straightforward variational argument with the ansatz $u(x, y, t)=w(t) \phi(x, y)$. We argue as follows: 


$$
\begin{aligned}
& \inf _{u \in C_{0}^{\infty}\left(\Pi_{v, d}^{+}\right)} \frac{\int_{\Pi_{v, d}^{+}}\left|\nabla_{\mathbb{H}^{n}} u\right|^{2} d \xi}{\int_{\Pi_{v, d}^{+}} \frac{|x|^{2}+|y|^{2}}{t^{2}}|u|^{2} d \xi} \leq \inf _{\substack{\phi \in C_{0}^{\infty}\left(\mathbb{R}^{2 n}\right) \\
w \in C_{0}^{\infty}\left(\mathbb{R}_{+}\right)}} \frac{\int_{\Pi_{v, d}^{+}\left|\nabla_{\mathbb{H}^{n}}(\phi w)\right|^{2} d \xi}}{\int_{\Pi_{v, d}^{+}} \frac{|x|^{2}+|y|^{2}}{t^{2}}|\phi w|^{2} d \xi} \\
& =\inf _{\substack{\phi \in C_{0}^{\infty}\left(\mathbb{R}^{2 n}\right) \\
w \in C_{0}^{\infty}\left(\mathbb{R}_{+}\right)}} \frac{\int_{\mathbb{R}_{+}} \int_{\mathbb{R}^{2 n}}\left(|w \nabla \phi|^{2}+4 w w^{\prime} \phi\left\langle\Lambda \xi^{\prime}, \nabla \phi\right\rangle+4\left|\xi^{\prime}\right|^{2}\left|\phi w^{\prime}\right|^{2}\right) d \xi^{\prime} d t}{\int_{\mathbb{R}_{+}} \int_{\mathbb{R}^{2 n}} \frac{|w|^{2}}{t^{2}}\left|\xi^{\prime}\right|^{2}|\phi|^{2} d \xi^{\prime} d t} \\
& =\inf _{\substack{\phi \in C_{0}^{\infty}\left(\mathbb{R}^{2 n}\right) \\
w \in C_{0}^{\infty}\left(\mathbb{R}_{+}\right)}}\left[\frac{\int_{\mathbb{R}_{+}}|w|^{2} d t}{\int_{\mathbb{R}_{+}} \frac{|w|^{2}}{t^{2}} d t} \cdot \frac{\int_{\mathbb{R}^{2 n}}|\nabla \phi|^{2} d \xi^{\prime}}{\int_{\mathbb{R}^{2 n}\left|\xi^{\prime}\right|^{2}|\phi|^{2} d \xi^{\prime}}}+4 \frac{\int_{\mathbb{R}_{+}} w w^{\prime} d t}{\int_{\mathbb{R}_{+}} \frac{|w|^{2}}{t^{2}} d t} \cdot \frac{\int_{\mathbb{R}^{2 n}} \phi\left\langle\Lambda \xi^{\prime}, \nabla \phi\right\rangle d \xi^{\prime}}{\int_{\mathbb{R}^{2 n}\left|\xi^{\prime}\right|^{2}|\phi|^{2} d \xi^{\prime}}}\right. \\
& \left.+4 \frac{\int_{\mathbb{R}_{+}}\left|w^{\prime}\right|^{2} d t}{\int_{\mathbb{R}_{+}} \frac{|w|^{2}}{t^{2}} d t} \cdot \frac{\int_{\mathbb{R}^{2 n}}\left|\xi^{\prime}\right|^{2}|\phi|^{2} d \xi^{\prime}}{\int_{\mathbb{R}^{2 n}}\left|\xi^{\prime}\right|^{2}|\phi|^{2} d \xi^{\prime}}\right] \\
& =\inf _{\substack{\phi \in C_{0}^{\infty}\left(\mathbb{R}^{2 n}\right) \\
w \in C_{0}^{\infty}\left(\mathbb{R}_{+}\right)}}\left[\frac{\int_{\mathbb{R}_{+}}|w|^{2} d t}{\int_{\mathbb{R}_{+}} \frac{|w|^{2}}{t^{2}} d t} \cdot \frac{\int_{\mathbb{R}^{2 n}}|\nabla \phi|^{2} d \xi^{\prime}}{\int_{\mathbb{R}^{2 n}\left|\xi^{\prime}\right|^{2}|\phi|^{2} d \xi^{\prime}}}+4 \frac{\int_{\mathbb{R}_{+}}\left|w^{\prime}\right|^{2} d t}{\int_{\mathbb{R}_{+}} \frac{|w|^{2}}{t^{2}} d t}\right]=1,
\end{aligned}
$$

where we used that $2 \int_{\mathbb{R}_{+}} w w^{\prime} d t=\int_{\mathbb{R}_{+}}\left(w^{2}\right)^{\prime} d t=0$, the sharp Hardy inequality on $\mathbb{R}_{+}$and that

$$
\inf _{\phi \in C_{0}^{\infty}\left(\mathbb{R}^{2 n}\right)} \frac{\int_{\mathbb{R}^{2 n}}|\nabla \phi|^{2} d \xi^{\prime}}{\int_{\mathbb{R}^{2 n}}\left|\xi^{\prime}\right|^{2}|\phi|^{2} d \xi^{\prime}}=0
$$

which follows by a simple dimensionality argument. Hence the constant appearing in Theorem 2.1 is sharp. For vertical planes, i.e. the ones that can not be reached by translation, the sharpness follows by a limiting procedure but can also be found by an almost identical variational argument but considering a slightly different quotient.

\subsection{An $L^{p}$ Hardy inequality on a half-space of $\mathbb{H}^{n}$}

We may use standard techniques to generalize the proof of the previous theorem to construct $L^{p}$ Hardy inequalities for any $p \geq 2$. We summarize the results in the following theorem.

Theorem 2.3 For $v \in \mathbb{S}^{2 n}$ and $d \in \mathbb{R}$ let $\Pi_{v, d}^{+}$be the half-space described previously. Then for $p \geq 2$ and $u \in C_{0}^{\infty}\left(\Pi_{v, d}^{+}\right)$the following inequality holds

$$
\int_{\Pi_{v, d}^{+}}\left|\nabla_{\mathbb{H}^{n}} u\right|^{p} d \xi \geq\left(\frac{p-1}{p}\right)^{p} \int_{\Pi_{v, d}^{+}} \sum_{i=1}^{n} \frac{\left|\left\langle X_{i}(\xi), v\right\rangle\right|^{p}+\left|\left\langle Y_{i}(\xi), v\right\rangle\right|^{p}}{\operatorname{dist}\left(\xi, \partial \Pi_{v, d}^{+}\right)^{p}}|u|^{p} d \xi
$$

Moreover, the constant in the inequality is sharp. 
The above $L^{p}$ version of our Hardy inequality on a half-space is perhaps not the most natural generalization of Theorem 2.1; a more natural weight in the right-hand side of (5) would be

$$
\frac{\left(\sum_{i=1}^{n}\left\langle X_{i}(\xi), v\right\rangle^{2}+\left\langle Y_{i}(\xi), v\right\rangle^{2}\right)^{p / 2}}{\operatorname{dist}\left(\xi, \partial \Pi_{v, d}^{+}\right)^{p}}
$$

However, by Jensen's inequality it is easy to see that Theorem 2.3 implies a Hardy inequality with the above weight but with a worse constant. We believe that such an inequality should hold with the same constant as in (5), namely $\left(\frac{p-1}{p}\right)^{p}$ (which is the sharp constant also for the Euclidean counterpart), but so far we are not able to prove this.

Proof of Theorem 2.3 The proof of the theorem is very similar to the proof presented above for the $L^{2}$ case. By the divergence theorem we have for $g \in H^{1}\left(\Pi_{\nu, d}^{+}\right)$and $V \in C^{\infty}\left(\Pi_{v, d}^{+} ; \mathbb{R}^{2 n+1}\right)$ that

$$
\int_{\Pi_{v, d}^{+}} \operatorname{div}(g V)|u|^{p} d \xi=-p \int_{\Pi_{v, d}^{+}} g\langle V, \nabla u\rangle \operatorname{sgn}(u)|u|^{p-1} d \xi .
$$

Here and in what follows div and $\nabla$ denote the usual divergence and gradient in $\mathbb{R}^{2 n+1}$. By Hölder's and Young's inequalities we have that

$$
\begin{aligned}
& -p \int_{\Pi_{v, d}^{+}} g\langle V, \nabla u\rangle \operatorname{sgn}(u)|u|^{p-1} d \xi \\
& \leq p\left(\int_{\Pi_{v, d}^{+}}|\langle V, \nabla u\rangle|^{p} d \xi\right)^{1 / p}\left(\int_{\Pi_{v, d}^{+}}|g|^{p /(p-1)}|u|^{p} d \xi\right)^{(p-1) / p} \\
& \leq \int_{\Pi_{v, d}^{+}}|\langle V, \nabla u\rangle|^{p} d \xi+(p-1) \int_{\Pi_{v, d}^{+}}|g|^{p /(p-1)}|u|^{p} d \xi
\end{aligned}
$$

Inserting this into (5) and rearranging the terms we obtain

$$
\int_{\Pi_{v, d}^{+}}\left(\operatorname{div}(g V)-(p-1)|g|^{p /(p-1)}\right)|u|^{p} d \xi \leq \int_{\Pi_{v, d}^{+}}|\langle V, \nabla u\rangle|^{p} d \xi .
$$

Choose $V=X_{i}$ and let

$$
g=\alpha \operatorname{sgn}\left(\left\langle X_{i}(\xi), \nu\right\rangle\right)\left(\frac{\left|\left\langle X_{i}(\xi), v\right\rangle\right|}{\operatorname{dist}\left(\xi, \partial \Pi_{\nu, d}^{+}\right)}\right)^{p-1} .
$$

By the same calculations as earlier we obtain that

$$
C(\alpha, p) \int_{\Pi_{v, d}^{+}} \frac{\left|\left\langle X_{i}(\xi), v\right\rangle\right|^{p}}{\operatorname{dist}\left(\xi, \partial \Pi_{v, d}^{+}\right)^{p}}|u|^{p} d \xi \leq \int_{\Pi_{v, d}^{+}}\left|X_{i} u\right|^{p} d \xi
$$


where

$$
C(\alpha, p)=-(p-1)\left(\alpha+|\alpha|^{p /(p-1)}\right) .
$$

Maximizing this constant in $\alpha$ we find that

$$
C(\alpha, p) \leq\left(\frac{p-1}{p}\right)^{p}
$$

where the maximum is attained at

$$
\alpha=-\left(\frac{p-1}{p}\right)^{p-1} .
$$

By an almost identical calculation but with $V=Y_{i}$ and

$$
g=\alpha \operatorname{sgn}\left(\left\langle Y_{i}(\xi), v\right\rangle\right)\left(\frac{\left|\left\langle Y_{i}(\xi), \nu\right\rangle\right|}{\operatorname{dist}\left(\xi, \partial \Pi_{\nu, d}^{+}\right)}\right)^{p-1}
$$

one finds that

$$
\left(\frac{p-1}{p}\right)^{p} \int_{\Pi_{v, d}^{+}} \frac{\left|\left\langle Y_{i}(\xi), v\right\rangle\right|^{p}}{\operatorname{dist}\left(\xi, \partial \Pi_{v, d}^{+}\right)^{p}}|u|^{p} d \xi \leq \int_{\Pi_{v, d}^{+}}\left|Y_{i} u\right|^{p} d \xi .
$$

Adding the two inequalities and summing over $i=1, \ldots, n$ we find that

$$
\begin{aligned}
& \left(\frac{p-1}{p}\right)^{p} \sum_{i=1}^{n} \int_{\Pi_{v, d}^{+}} \frac{\left|\left\langle X_{i}(\xi), v\right\rangle\right|^{p}+\left|\left\langle Y_{i}(\xi), v\right\rangle\right|^{p}}{\operatorname{dist}\left(\xi, \partial \Pi_{\nu, d}^{+}\right)^{p}}|u|^{p} d \xi \\
& \leq \int_{\Pi_{v, d}^{+}} \sum_{i=1}^{n}\left(\left|X_{i} u\right|^{p}+\left|Y_{i} u\right|^{p}\right) d \xi .
\end{aligned}
$$

Since $p \geq 2$, the function $\varphi: x \mapsto x^{p / 2}$ is superadditive, and therefore

$$
\begin{aligned}
& \sum_{i=1}^{n}\left(\left|X_{i} u\right|^{p}+\left|Y_{i} u\right|^{p}\right)=\sum_{i=1}^{n}\left(\left|X_{i} u\right|^{2}\right)^{p / 2}+\left(\left|Y_{i} u\right|^{2}\right)^{p / 2} \leq\left(\sum_{i=1}^{n}\left|X_{i} u\right|^{2}+\left|Y_{i} u\right|^{2}\right)^{p / 2} \\
& \quad=\left|\nabla_{\mathbb{H}^{n}} u\right|^{p}
\end{aligned}
$$

Inserting this into the above we get (5).

By a similar argument as in the case $p=2$ we can prove that the constant is sharp in the sense that if it were replaced by a larger constant we could choose $\Pi_{v, d}^{+}$such that the inequality fails. To achieve this we wish to find an upper bound for the quantity

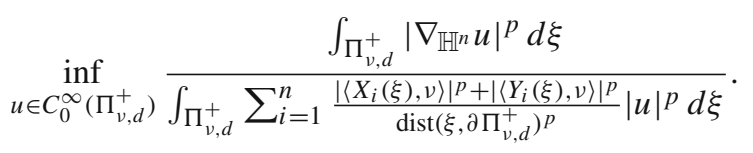


We begin by choosing $v_{0}=(1,0, \ldots, 0)$ and $d=0$. By the same calculations as in the $L^{2}$ case we then find that the quotient can be rewritten in the form

$$
\inf _{u \in C_{0}^{\infty}\left(\Pi_{\nu_{0}}^{+}\right)} \frac{\int_{\Pi_{v_{0}}^{+}}\left(\left|\nabla^{\prime} u\right|^{2}+4 \frac{\partial u}{\partial t}\left\langle\Lambda \xi^{\prime}, \nabla^{\prime} u\right\rangle+4\left|\xi^{\prime}\right|^{2}\left|\frac{\partial u}{\partial t}\right|^{2}\right)^{p / 2} d \xi}{\int_{\Pi_{\nu_{0}}^{+}} \frac{|u|^{p}}{x_{1}^{p}} d \xi}
$$

With the same ansatz as before, namely $u(\xi)=\phi\left(\xi^{\prime}\right) w(t)$, we can bound this from above by

$$
\inf _{\substack{\phi \in C_{0}^{\infty}\left(\mathbb{R}_{+}^{2 n}\right) \\ w \in C_{0}^{\infty}(\mathbb{R})}} \frac{\int_{\mathbb{R}_{+}^{2 n}} \int_{\mathbb{R}}\left(|\nabla \phi|^{2}|w|^{2}+4\left|w w^{\prime}\right|\left|\phi\left\langle\Lambda \xi^{\prime}, \nabla \phi\right\rangle\right|+4\left|\xi^{\prime}\right|^{2}|\phi|^{2}\left|w^{\prime}\right|^{2}\right)^{p / 2} d t d \xi^{\prime}}{\int_{\mathbb{R}_{+}^{2 n}} \int_{\mathbb{R}} \frac{|\phi w|^{p}}{x_{1}^{p}} d t d \xi^{\prime}},
$$

where $\mathbb{R}_{+}^{2 n}=\left\{\xi^{\prime} \in \mathbb{R}^{2 n}: x_{1}>0\right\}$.

In the case $p=2$ things are slightly simpler and the above quotient splits into three parts, one of which easily can be seen to be zero and another which can be eliminated by a simple scaling argument. However, in the general case we cannot in such a simple manner split the above integral. But using Jensen's inequality we can bound the quotient by some appropriately weighted sum of three terms and then use a similar scaling argument as for $p=2$.

What we need is the following simple consequence of Jensen's inequality: For $\alpha \geq 1, x_{i} \geq 0$ and any $a_{i}>0, i=1, \ldots, k$, we have that

$$
\begin{aligned}
\left(\sum_{i=1}^{k} x_{i}\right)^{\alpha} & =\left(\sum_{i=1}^{k} a_{i}\right)^{\alpha}\left(\frac{\sum_{i=1}^{k} a_{i}\left(x_{i} / a_{i}\right)}{\sum_{i=1}^{k} a_{i}}\right)^{\alpha} \\
& \leq\left(\sum_{i=1}^{k} a_{i}\right)^{\alpha}\left(\frac{\sum_{i=1}^{k} a_{i}\left(x_{i} / a_{i}\right)^{\alpha}}{\sum_{i=1}^{k} a_{i}}\right) \\
& =\sum_{i=1}^{k} a_{i}^{1-\alpha}\left(\sum_{j=1}^{k} a_{j}\right)^{\alpha-1} x_{i}^{\alpha} .
\end{aligned}
$$

We will apply this with $k=3, \alpha=p / 2, x_{1}=|\nabla \phi|^{2}|w|^{2}, x_{2}=4\left|w w^{\prime}\right|\left|\phi\left\langle\Lambda \xi^{\prime}, \nabla \phi\right\rangle\right|$, $x_{3}=4\left|\xi^{\prime}\right|^{2}|\phi|^{2}\left|w^{\prime}\right|^{2}$ and $a_{i}$ 's to be chosen later. We also denote the effective weights of each $x_{i}$ by $c_{i}$, that is

$$
c_{i}=a_{i}^{1-p / 2}\left(\sum_{j=1}^{3} a_{j}\right)^{p / 2-1} .
$$


Using the above we find that

$$
\begin{aligned}
& \inf _{u \in C_{0}^{\infty}} \frac{\int_{\Pi_{\nu_{0}}^{+}}\left|\nabla_{\mathbb{H}^{n}} u\right|^{p} d \xi}{\int_{\Pi_{\nu_{0}}^{+}} \frac{|u|^{p}}{x_{1}^{p}} d \xi}=\inf _{u \in C_{0}^{\infty}} \frac{\int_{\Pi_{\nu_{0}}^{+}}\left(\left|\nabla^{\prime} u\right|^{2}+4 \frac{\partial u}{\partial t}\left\langle\Lambda \xi^{\prime}, \nabla^{\prime} u\right\rangle+4\left|\xi^{\prime}\right|^{2}\left|\frac{\partial u}{\partial t}\right|^{2}\right)^{p / 2} d \xi}{\int_{\Pi_{\nu_{0}}^{+}} \frac{|u|^{p}}{x_{1}^{p}} d \xi} \\
& \leq \inf _{\substack{\phi \in C_{0}^{\infty} \\
w \in C_{0}^{\infty}}} \frac{\int_{\mathbb{R}_{+}^{2 n}}^{2 n} \int_{\mathbb{R}}\left(c_{1}|\nabla \phi|^{p}|w|^{p}+2^{p} c_{2}\left|w w^{\prime}\right|^{p / 2}\left|\phi\left\langle\Lambda \xi^{\prime}, \nabla \phi\right\rangle\right|^{p / 2}+2^{p} c_{3}\left|\xi^{\prime}\right|^{p}|\phi|^{p}\left|w^{\prime}\right|^{p}\right) d t d \xi^{\prime}}{\int_{\mathbb{R}_{+}^{2 n}} \int_{\mathbb{R}} \frac{\mid \phi w p^{p}}{x_{1}^{p}} d t d \xi^{\prime}} \\
& =\inf _{\phi \in C_{0}^{\infty}}\left[c_{1} \frac{\int_{\mathbb{R}_{+}^{2 n}}|\nabla \phi|^{p} d \xi^{\prime}}{\int_{\mathbb{R}_{+}^{2 n}} \frac{|\phi|^{p}}{x_{1}^{p}} d \xi^{\prime}} \cdot \frac{\int_{\mathbb{R}}|w|^{p} d t}{\int_{\mathbb{R}}|w|^{p} d t}\right. \\
& \left.+2^{p} C_{0}^{\infty} \frac{\int_{\mathbb{R}_{+}^{2 n}}\left|\phi\left\langle\Lambda \xi^{\prime}, \nabla \phi\right\rangle\right|^{p / 2} d \xi^{\prime}}{\int_{\mathbb{R}_{+}^{2 n}} \frac{|\phi|^{p}}{x_{1}^{p}} d \xi^{\prime}} \cdot \frac{\int_{\mathbb{R}}\left|w w^{\prime}\right|^{p / 2} d t}{\int_{\mathbb{R}}|w|^{p} d t}+2^{p} c_{3} \frac{\int_{\mathbb{R}_{+}^{2 n}}\left|\xi^{\prime}\right|^{p}|\phi|^{p} d \xi^{\prime}}{\int_{\mathbb{R}_{+}^{2 n}} \frac{|\phi|^{p}}{x_{1}^{p}} d \xi^{\prime}} \cdot \frac{\int_{\mathbb{R}}\left|w^{\prime}\right|^{p} d t}{\int_{\mathbb{R}}|w|^{p} d t}\right] .
\end{aligned}
$$

By a simple rescaling argument in $t$, by say replacing $w(t)$ by $\widetilde{w}(t)=w(\lambda t)$ with $\lambda>0$, one sees that we independently of choice of $\phi$ can make the last two terms arbitrarily small. Moreover, since the first term is independent of $w$ we can use the sharp Hardy inequality in $\mathbb{R}_{+}^{2 n}$ (see, for instance, $[13,18]$ ) to find that

$$
\inf _{u \in W_{0}^{1, p}} \frac{\int_{\Pi_{v_{0}}^{+}} \mid \nabla_{\left.\mathbb{H}^{n} u\right|^{p}} d \xi}{\int_{\Pi_{v_{0}}^{+}} \frac{|u|^{p}}{x_{1}^{p}} d \xi} \leq c_{1}\left(\frac{p-1}{p}\right)^{p}
$$

Since we have lost all dependence on $c_{2}$ and $c_{3}$ we are free to choose the $a_{i}$ in such a way that $c_{1}$ is arbitrarily close to 1 , which can be done by fixing $a_{2}, a_{3}$ positive and choosing $a_{1}$ sufficiently large. This completes the proof.

\section{Hardy inequalities for convex domains in $\mathbb{H}^{n}$}

We now turn our attention to using Theorem 2.1 to obtain a geometric version of Hardy's inequality on convex domains in $\mathbb{H}^{n}$.

Theorem 3.1 Let $\Omega$ be a convex domain in $\mathbb{H}^{n}$. For $\xi \in \Omega$ let $v(\xi)$ denote the unit normal of $\partial \Omega$ at a point $\hat{\xi} \in \partial \Omega$, where $\hat{\xi}$ is such that $\operatorname{dist}(\xi, \partial \Omega)=\operatorname{dist}(\hat{\xi}, \xi)$. Then for any $u \in C_{0}^{\infty}(\Omega)$ we have that

$$
\int_{\Omega}\left|\nabla_{\mathbb{H}^{n}} u\right|^{2} d \xi \geq \frac{1}{4} \int_{\Omega} \sum_{i=1}^{n} \frac{\left\langle X_{i}(\xi), v(\xi)\right\rangle^{2}+\left\langle Y_{i}(\xi), v(\xi)\right\rangle^{2}}{\operatorname{dist}(\xi, \partial \Omega)^{2}}|u|^{2} d \xi
$$

Note that we do not require the domain $\Omega$ to be bounded. In particular if $\Omega$ is a half-space of $\mathbb{H}^{n}$ this is precisely Theorem 2.1. The proof is based on an approach used in [3] and proceeds along the same lines as the that of Theorem 2.1 with an additional element in which we approximate the domain $\Omega$ by convex polytopes. 
Proof of Theorem 3.1 We begin by proving the inequality when $\Omega$ is a convex polytope. Let $\left\{F_{k}\right\}_{k}$ be the facets of $\Omega$ with corresponding inward pointing unit normals $\left\{v_{k}\right\}_{k}$. Further we construct a partition of $\Omega$ into the essentially disjoint sets $\Omega_{k}:=\left\{\xi \in \Omega: \operatorname{dist}(\xi, \partial \Omega)=\operatorname{dist}\left(\xi, F_{k}\right)\right\}$. Since the partition elements $\Omega_{k}$ are defined through a finite number of affine inequalities they are polytopes.

For each partition element $\Omega_{k}$ we can now apply the same idea as in the proof of the previous theorem. The only difference will be that not all the boundary terms from the partial integration are zero. In each $\Omega_{k}$ we define the potential $V$ with components

$$
\begin{aligned}
V_{i}(\xi) & =\frac{\left\langle X_{i}(\xi), v_{k}\right\rangle}{\operatorname{dist}\left(\xi, F_{k}\right)}, \\
V_{n+i}(\xi) & =\frac{\left\langle Y_{i}(\xi), v_{k}\right\rangle}{\operatorname{dist}\left(\xi, F_{k}\right)} .
\end{aligned}
$$

Through the same calculations as before one finds that

$$
\begin{aligned}
0 \leq & \sum_{i=1}^{n} \int_{\Omega_{k}}\left(\left|\left(X_{i}+\alpha V_{i}\right) u\right|^{2}+\left|\left(Y_{i}+\alpha V_{n+i}\right) u\right|^{2}\right) d \xi \\
= & \sum_{i=1}^{n} \int_{\Omega_{k}}\left(\left|X_{i} u\right|^{2}+\left|Y_{i} u\right|^{2}-\alpha|u|^{2}\left(X_{i}\left(V_{i}\right)+Y_{i}\left(V_{n+i}\right)\right)+\alpha^{2}|u|^{2}\left(V_{i}^{2}+V_{n+i}^{2}\right)\right) d \xi \\
& +\alpha \sum_{i=1}^{n} \int_{\partial \Omega_{k}}|u|^{2}\left(V_{i}\left\langle X_{i}(\xi), n_{k}(\xi)\right\rangle+V_{n+i}\left\langle Y_{i}(\xi), n_{k}(\xi)\right\rangle\right) d \Gamma_{\partial \Omega_{k}}(\xi),
\end{aligned}
$$

where $n_{k}(\xi)$ denotes the outward pointing unit normal of $\partial \Omega_{k}$ at $\xi$. Note that on $F_{k} \subset \partial \Omega_{k}$ we have that $n_{k}(\xi)=-v_{k}$.

Since $u$ is compactly supported in $\Omega$ the boundary contribution from $\partial \Omega$ is again zero, and thus all we need to deal with are the parts of $\partial \Omega_{k}$ that are in the interior of $\Omega$. For each such facet of $\Omega_{k}$ there is some $\Omega_{l}, l \neq k$, that shares this facet. Let $\Gamma_{k l}$ denote the common facet of $\Omega_{k}$ and $\Omega_{l}$, and note that $\left.n_{k}\right|_{\Gamma_{k l}}=-\left.n_{l}\right|_{\Gamma_{k l}}$. Summing over all partition elements $\Omega_{k}$ and letting $n_{k l}=\left.n_{k}\right|_{\Gamma_{k l}}$, i.e. the unit normal of $\Gamma_{k l}$ pointing from $\Omega_{k}$ into $\Omega_{l}$, we obtain using the earlier calculations for the components of $V$ that

$$
\begin{aligned}
0 \leq & \int_{\Omega}\left|\nabla_{\mathbb{H}^{n}} u\right|^{2} d \xi-\frac{1}{4} \int_{\Omega} \sum_{i=1}^{n} \frac{\left\langle X_{i}(\xi), v(\xi)\right\rangle^{2}+\left\langle Y_{i}(\xi), v(\xi)\right\rangle^{2}}{\operatorname{dist}(\xi, \partial \Omega)^{2}}|u|^{2} d \xi \\
& -\frac{1}{2} \sum_{k \neq l} \sum_{i=1}^{n} \int_{\Gamma_{k l}} \frac{\left\langle X_{i}(\xi), v_{k}\right\rangle\left\langle X_{i}(\xi), n_{k l}\right\rangle+\left\langle Y_{i}(\xi), v_{k}\right\rangle\left\langle Y_{i}(\xi), n_{k l}\right\rangle}{\operatorname{dist}\left(\xi, F_{k}\right)}|u|^{2} d \Gamma_{k l} \\
= & \int_{\Omega}\left|\nabla_{\mathbb{H}^{n}} u\right|^{2} d \xi-\frac{1}{4} \int_{\Omega} \sum_{i=1}^{n} \frac{\left\langle X_{i}(\xi), v(\xi)\right\rangle^{2}+\left\langle Y_{i}(\xi), v(\xi)\right\rangle^{2}}{\operatorname{dist}(\xi, \partial \Omega)^{2}}|u|^{2} d \xi \\
& -\frac{1}{2} \sum_{k<l} \sum_{i=1}^{n} \int_{\Gamma_{k l}} \frac{\left\langle X_{i}(\xi), v_{k}-v_{l}\right\rangle\left\langle X_{i}(\xi), n_{k l}\right\rangle+\left\langle Y_{i}(\xi), v_{k}-v_{l}\right\rangle\left\langle Y_{i}(\xi), n_{k l}\right\rangle}{\operatorname{dist}\left(\xi, F_{k}\right)}|u|^{2} d \Gamma_{k l} .
\end{aligned}
$$


In the last equality we use the fact that $\Gamma_{k l}$ is by definition the set where $\operatorname{dist}\left(\xi, F_{k}\right)=$ $\operatorname{dist}\left(\xi, F_{l}\right)$.

By construction we have that

$$
\Gamma_{k l}=\left\{\xi: \xi \cdot v_{k}-d_{k}=\xi \cdot v_{l}-d_{l}\right\} .
$$

Rearranging we find that $\xi \cdot\left(v_{k}-v_{l}\right)-d_{k}+d_{l}=0$, that is, $\Gamma_{k l}$ is a hyperplane with normal $v_{k}-v_{l}$. Therefore, we have $v_{k}-v_{l} \| n_{k l}$ and all that remains to do is check that $\left(v_{k}-v_{l}\right) \cdot n_{k l}>0$. Since $v_{k}$ points into $k$-th partition element and $n_{k l}$ points out, $v_{k} \cdot n_{k l}$ is non-negative. By the same argument the term $v_{l} \cdot n_{k l}$ is non-positive. Therefore, the entire expression is non-negative and moreover we have that

$$
\begin{aligned}
\left|v_{k}-v_{l}\right|^{2}=\left(v_{k}-v_{l}\right) \cdot\left(v_{k}-v_{l}\right) & =2-2 v_{k} \cdot v_{l} \\
& =2-2 \cos \left(\alpha_{k l}\right),
\end{aligned}
$$

where $\alpha_{k l}$ is the angle between $v_{k}$ and $v_{l}$. Thus $\left(v_{k}-v_{l}\right) \cdot n_{k l}=\sqrt{2-2 \cos \alpha_{k l}}$ and equation (6) gives us that

$$
\begin{aligned}
0 \leq & \int_{\Omega}\left|\nabla_{\mathbb{H}^{n}} u\right|^{2} d \xi-\frac{1}{4} \int_{\Omega} \sum_{i=1}^{n} \frac{\left\langle X_{i}(\xi), v(\xi)\right\rangle^{2}+\left\langle Y_{i}(\xi), v(\xi)\right\rangle^{2}}{\operatorname{dist}(\xi, \partial \Omega)^{2}}|u|^{2} d \xi \\
& -\frac{1}{\sqrt{2}} \sum_{k<l} \sum_{i=1}^{n} \int_{\Gamma_{k l}} \sqrt{1-\cos \alpha_{k l}} \frac{\left\langle X_{i}(\xi), n_{k l}\right\rangle^{2}+\left\langle Y_{i}(\xi), n_{k l}\right\rangle^{2}}{\operatorname{dist}\left(\xi, F_{k}\right)}|u|^{2} d \Gamma_{k l} .
\end{aligned}
$$

We conclude that the boundary terms are of the correct sign, and the inequality follows.

Let now $\Omega$ be an arbitrary convex domain. For $u \in C_{0}^{\infty}(\Omega)$ we can always choose an increasing sequence of convex polytopes $\left\{\Omega_{j}\right\}_{j=1}^{\infty}$ such that $u \in C_{0}^{\infty}\left(\Omega_{1}\right), \Omega_{j} \subset \Omega$ and $\Omega_{j} \rightarrow \Omega$ when $j \rightarrow \infty$. Letting $v_{j}(\xi)$ be the map $v$ from above corresponding to $\Omega_{j}$ we have that

$$
\begin{aligned}
\int_{\Omega}\left|\nabla_{\mathbb{H}^{n}} u\right|^{2} d \xi & =\int_{\Omega_{j}}\left|\nabla_{\mathbb{H}^{n}} u\right|^{2} d \xi \\
& \geq \frac{1}{4} \int_{\Omega_{j}} \sum_{i=1}^{n} \frac{\left\langle X_{i}(\xi), v_{j}(\xi)\right\rangle^{2}+\left\langle Y_{i}(\xi), v_{j}(\xi)\right\rangle^{2}}{\operatorname{dist}\left(\xi, \partial \Omega_{j}\right)^{2}}|u|^{2} d \xi \\
& =\frac{1}{4} \int_{\Omega} \sum_{i=1}^{n} \frac{\left\langle X_{i}(\xi), v_{j}(\xi)\right\rangle^{2}+\left\langle Y_{i}(\xi), v_{j}(\xi)\right\rangle^{2}}{\operatorname{dist}\left(\xi, \partial \Omega_{j}\right)^{2}}|u|^{2} d \xi \\
& \geq \frac{1}{4} \int_{\Omega} \sum_{i=1}^{n} \frac{\left\langle X_{i}(\xi), v_{j}(\xi)\right\rangle^{2}+\left\langle Y_{i}(\xi), v_{j}(\xi)\right\rangle^{2}}{\operatorname{dist}(\xi, \partial \Omega)^{2}}|u|^{2} d \xi
\end{aligned}
$$

Letting $j$ tend to infinity completes the proof. 


\section{1 $L^{p}$ Hardy inequality for a convex domain}

Again we can make slight alterations to the proof above to obtain $L^{p}$-inequalities, $p \geq 2$, on convex domains. We summarize the results in the following theorem.

Theorem 3.2 Let $\Omega$ be a convex domain in $\mathbb{H}^{n}$ and for $\xi \in \Omega$ let $v(\xi)$ denote the unit normal of $\partial \Omega$ at a point $\hat{\xi} \in \partial \Omega$, where $\hat{\xi}$ is such that $\operatorname{dist}(\xi, \partial \Omega)=\operatorname{dist}(\hat{\xi}, \xi)$. Then for any $p \geq 2$ and $u \in C_{0}^{\infty}(\Omega)$ the following inequality holds

$$
\int_{\Omega}\left|\nabla_{\mathbb{H}^{n}} u\right|^{p} d \xi \geq\left(\frac{p-1}{p}\right)^{p} \int_{\Omega} \sum_{i=1}^{n} \frac{\left|\left\langle X_{i}(\xi), v(\xi)\right\rangle\right|^{p}+\left|\left\langle Y_{i}(\xi), v(\xi)\right\rangle\right|^{p}}{\operatorname{dist}(\xi, \partial \Omega)^{p}}|u|^{p} d \xi
$$

Proof of Theorem 3.2 The proof of the theorem is very similar to the proof presented above for the $L^{2}$ case. We again begin with the case when $\Omega$ is a polytope and consider the same partition $\Omega_{k}$. For $g \in H^{1}\left(\Omega_{k}\right)$ and $V \in C^{\infty}\left(\Omega_{k} ; \mathbb{R}^{2 n+1}\right)$ the divergence theorem gives us that

$$
\begin{aligned}
\int_{\Omega_{k}} \operatorname{div}(g V)|u|^{p} d \xi= & -p \int_{\Omega_{k}} g\langle V, \nabla u\rangle \operatorname{sgn}(u)|u|^{p-1} d \xi \\
& +\int_{\partial \Omega_{k}} g\left\langle V, n_{k}(\xi)\right\rangle|u|^{p} d \Gamma_{\partial \Omega_{k}}(\xi) .
\end{aligned}
$$

Consider the first term in the right-hand side. Using Hölder's and Young's inequalities we have that

$$
\begin{aligned}
& -p \int_{\Omega_{k}} g\langle V, \nabla u\rangle \operatorname{sgn}(u)|u|^{p-1} d \xi \\
& \leq p\left(\int_{\Omega_{k}}|\langle V, \nabla u\rangle|^{p} d \xi\right)^{1 / p}\left(\int_{\Omega_{k}}|g|^{p /(p-1)}|u|^{p} d \xi\right)^{(p-1) / p} \\
& \leq \int_{\Omega_{k}}|\langle V, \nabla u\rangle|^{p} d \xi+(p-1) \int_{\Omega_{k}}|g|^{p /(p-1)}|u|^{p} d \xi .
\end{aligned}
$$

Inserting this into 3.1 and rearranging the terms we obtain

$$
\begin{aligned}
\int_{\Omega_{k}}\left(\operatorname{div}(g V)-(p-1)|g|^{p /(p-1)}\right)|u|^{p} d \xi & \leq \int_{\Omega_{k}}|\langle V, \nabla u\rangle|^{p} d \xi \\
& +\int_{\partial \Omega_{k}} g\left\langle V, n_{k}(\xi)\right\rangle|u|^{p} d \Gamma_{\partial \Omega_{k}}(\xi) .
\end{aligned}
$$

Choosing $V=X_{i}$ and letting

$$
g=\alpha \operatorname{sgn}\left(\left\langle X_{i}(\xi), v_{k}\right\rangle\right)\left(\frac{\left|\left\langle X_{i}(\xi), v_{k}\right\rangle\right|}{\operatorname{dist}\left(\xi, F_{k}\right)}\right)^{p-1}
$$


we see by the same calculations as earlier that

$$
\begin{aligned}
& C(\alpha, p) \int_{\Omega_{k}} \frac{\left\langle X_{i}(\xi), v_{k}\right\rangle^{p}}{\operatorname{dist}\left(\xi, F_{k}\right)^{p}}|u|^{p} d \xi \leq \int_{\Omega_{k}}\left|X_{i} u\right|^{p} d \xi \\
& \quad+\alpha \int_{\partial \Omega_{k}} \operatorname{sgn}\left(\left\langle X_{i}(\xi), v_{k}\right\rangle\right)\left(\frac{\left|\left\langle X_{i}(\xi), v_{k}\right\rangle\right|}{\operatorname{dist}\left(\xi, F_{k}\right)}\right)^{p-1}\left\langle X_{i}(\xi), n_{k}(\xi)\right\rangle|u|^{p} d \Gamma_{\partial \Omega_{k}}(\xi)
\end{aligned}
$$

where

$$
C(\alpha, p)=-(p-1)\left(\alpha-|\alpha|^{p /(p-1)}\right) .
$$

Maximizing this constant in $\alpha$ we find as before that

$$
C(\alpha, p) \leq\left(\frac{p-1}{p}\right)^{p},
$$

where the maximum is attained at

$$
\alpha=-\left(\frac{p-1}{p}\right)^{p-1} .
$$

What remains to complete the proof is to show that we can discard the boundary terms after summing over the $\Omega_{k}$, in other words we need to prove that

$$
\begin{aligned}
& -\left(\frac{p-1}{p}\right)^{p-1} \sum_{k} \int_{\partial \Omega_{k}} \operatorname{sgn}\left(\left\langle X_{i}(\xi), v_{k}\right\rangle\right)\left(\frac{\left|\left\langle X_{i}(\xi), v_{k}\right\rangle\right|}{\operatorname{dist}\left(\xi, F_{k}\right)}\right)^{p-1} \\
& \left\langle X_{i}(\xi), n_{k}(\xi)\right\rangle|u|^{p} d \Gamma_{\partial \Omega_{k}}(\xi) \leq 0 .
\end{aligned}
$$

As in the $L^{2}$ case only the boundary terms that come from the interior of $\Omega$ are nonzero, and again these appear in pairs. Thus, in the same manner as before we wish to show that

$$
\begin{aligned}
& \int_{\Gamma_{k l}}\left[\operatorname{sgn}\left(\left\langle X_{i}(\xi), v_{k}\right\rangle\right)\left(\frac{\left|\left\langle X_{i}(\xi), v_{k}\right\rangle\right|}{\operatorname{dist}\left(\xi, F_{k}\right)}\right)^{p-1}\left\langle X_{i}(\xi), n_{k l}\right\rangle\right. \\
& \left.-\operatorname{sgn}\left(\left\langle X_{i}(\xi), v_{l}\right\rangle\right)\left(\frac{\left|\left\langle X_{i}(\xi), v_{l}\right\rangle\right|}{\operatorname{dist}\left(\xi, F_{l}\right)}\right)^{p-1}\left\langle X_{i}(\xi), n_{k l}\right\rangle\right]|u|^{p} d \Gamma_{k l}
\end{aligned}
$$

is non-negative.

By the same geometric considerations as above $n_{k l}=\beta\left(v_{k}-v_{l}\right)$, where $\beta=$ $1 / \sqrt{2-2 \cos \left(\alpha_{k l}\right)}$. Using this combined with the fact that $\operatorname{dist}\left(\xi, F_{k}\right)=\operatorname{dist}\left(\xi, F_{l}\right)$ on $\Gamma_{k l}$ we find that (3.1) can be rewritten as 


$$
\begin{aligned}
& \beta \int_{\Gamma_{k l}}\left[\left|\left\langle X_{i}(\xi), v_{k}\right\rangle\right|^{p}-\operatorname{sgn}\left(\left\langle X_{i}(\xi), v_{k}\right\rangle\right)\left|\left\langle X_{i}(\xi), v_{k}\right\rangle\right|^{p-1}\left\langle X_{i}(\xi), v_{l}\right\rangle+\left|\left\langle X_{i}(\xi), v_{l}\right\rangle\right|^{p}\right. \\
& \left.\quad-\operatorname{sgn}\left(\left\langle X_{i}(\xi), v_{l}\right\rangle\right)\left|\left\langle X_{i}(\xi), v_{l}\right\rangle\right|^{p-1}\left\langle X_{i}(\xi), v_{k}\right\rangle\right] \frac{|u|^{p}}{\operatorname{dist}\left(\xi, F_{k}\right)^{p-1}} d \Gamma_{k l} .
\end{aligned}
$$

Thus it suffices to proof that the expression in the brackets is positive. Clearly this is positive when $\left\langle X_{i}(\xi), v_{k}\right\rangle$ and $\left\langle X_{i}(\xi), v_{l}\right\rangle$ have different signs. On the other hand if the two scalar products have the same sign we have an expression of the form $A^{p}-A^{p-1} B-B^{p-1} A+B^{p-1}$, where $A=\left|\left\langle X_{i}(\xi), v_{k}\right\rangle\right|$ and $B=\left|\left\langle X_{i}(\xi), v_{l}\right\rangle\right|$. But this we can rewrite as

$$
A^{p}-A^{p-1} B-B^{p-1} A+B^{p-1}=\left(A^{p-1}-B^{p-1}\right)(A-B) .
$$

Now it is clear that both terms on the right-hand side have the same sign. Thus (7) is non-negative and we conclude that the boundary terms can be discarded.

By almost identical calculations we find the corresponding inequalities for the $Y_{i}$. Summing all terms and using Jensen's inequality as in the proof of Theorem 2.3 we obtain the desired inequality for polytopes. The proof of the theorem can now be completed in the same manner as for Theorem 3.1.

Acknowledgments It is a pleasure to thank Professor Ari Laptev for suggesting the problem studied here and for his input and encouragement. Part of this work was carried out during a visit to the Institute of Analysis, Dynamics and Modelling at Universität Stuttgart and the author would like to thank the staff for their hospitality, and in particular Professor Timo Weidl for the invitation. The author would also like to thank Eric Larsson, Bartosch Ruszkowski and Aron Wennman for fruitful discussions. The author is supported by the Swedish Research Council Grant No. 2012-3864.

Open Access This article is distributed under the terms of the Creative Commons Attribution 4.0 International License (http://creativecommons.org/licenses/by/4.0/), which permits unrestricted use, distribution, and reproduction in any medium, provided you give appropriate credit to the original author(s) and the source, provide a link to the Creative Commons license, and indicate if changes were made.

\section{References}

1. Arcozzi, N., Ferrari, F.: Metric normal and distance function in the Heisenberg group. Math. Z. 256(3), 661-684 (2007)

2. Arcozzi, N., Ferrari, F.: The Hessian of the distance from a surface in the Heisenberg group. Ann. Acad. Sci. Fenn. Math. 33(1), 35-63 (2008)

3. Avkhadiev, F.G.: Hardy type inequalities in higher dimensions with explicit estimate of constants. Lobachevskii J. Math. 21, 3-31 (2006)

4. Balinsky, A.A., Evans, W.D., Lewis, R.T.: The Analysis and Geometry of Hardy's Inequality. Universitext, Springer International Publishing, New York (2015)

5. Chow, W.L.: Über Systeme von linearen partiellen Differentialgleichungen erster Ordnung. Math. Ann. 117, 98-105 (1939)

6. D'Ambrosio, L.: Some hardy inequalities on the Heisenberg group. Differ. Equ. 40(4), 552-564 (2004)

7. Danielli, D., Garofalo, N., Phuc, N.C.: Inequalities of Hardy-Sobolev type in Carnot-Carathéodory spaces, Sobolev spaces in mathematics. I Int. Math. Ser. (N. Y.), vol. 8, pp. 117-151. Springer, New York (2009)

8. Davies, E.B.: Heat kernels and Spectral Theory, Cambridge Tracts in Mathematics, vol. 92. Cambridge Univ. Press, Cambridge (1989) 
9. Garofalo, N., Lanconelli, E.: Frequency functions on the Heisenberg group, the uncertainty principle and unique continuation. Ann. Inst. Fourier (Grenoble) 40(2), 313-356 (1990)

10. Hörmander, L.: Hypoelliptic second order differential equations. Acta Math. 119(1), 147-171 (1967)

11. Jin, Y:: Hardy-type inequalities on H-type groups and anisotropic Heisenberg groups. Chin. Ann. Math. Ser. B 29(5), 567-574 (2008)

12. Luan, J., Yang, Q.: A Hardy type inequality in the half-space on $\mathbb{R}^{n}$ and Heisenberg group. J. Math. Anal. Appl. 347(2), 645-651 (2008)

13. Maz'ya, V.: Sobolev spaces with applications to elliptic partial differential equations, augmented ed., Fundamental Principles of Mathematical Sciences. Springer, Heidelberg (2011)

14. Prandi, D., Rizzi, L., Seri, M.: A sub-Riemannian Santaló formula with applications to isoperimetric inequalities and first Dirichlet eigenvalue of hypoelliptic operators, p. 1509.05415. ArXiv e-prints, (2015)

15. Rashevsky, P.K.: Any two points of a totally nonholonomic space may be connected by an admissible line. Uch. Zap. Ped. Inst. Liebknechta Ser. Phys. Math. 2, 83-94 (1938). Russian

16. Ruszkowski, B.: Hardy inequalities for the Heisenberg Laplacian on convex bounded polytopes, in preperation (2016)

17. Ruzhansky, M., Suragan, D.: Local Hardy inequality for sums of squares. ArXiv e-prints, 1601.06157 (2016)

18. Tidblom, J.: A Hardy inequality in the half-space. J. Funct. Anal. 221(2), 482-495 (2005) 